\title{
ON COMPUTING LINEARIZING COORDINATES FROM SYMMETRY ALGEBRA
}

\author{
Sajid Ali ${ }^{1}$, Hassan Azad ${ }^{2}$, Waqas Shah ${ }^{2}$, and Fazal Mahomed ${ }^{3}$ \\ ${ }^{1}$ Zayed University \\ ${ }^{2}$ Abdus Salam School of Mathematical Sciences \\ ${ }^{3}$ University of the Witwatersrand
}

June 13, 2020

\begin{abstract}
A characterization of the symmetry algebra of the $\$ \mathrm{~N} \$$ th-order ordinary differential equations (ODEs) with maximal symmetry and all third-order linearizable ODEs is given. This is used to show that such an algebra $\$ \backslash \operatorname{mathfrak}\{\mathrm{g}\} \$$ determines - up to a point transformation - only one linear equation whose symmetry algebra is $\$ \backslash$ mathfrak $\{\mathrm{g}\} \$$ and an algorithmic procedure is given to find the linearizing coordinates. The procedure is illustrated by several examples from the literature.
\end{abstract}

\section{Hosted file}

linearization coordinates-rev.pdf available at https://authorea.com/users/333077/articles/ 459354-on-computing-linearizing-coordinates-from-symmetry-algebra 